\title{
Editorial \\ TOPOLOGICAL AND VARIATIONAL METHODS OF NONLINEAR ANALYSIS AND THEIR APPLICATIONS
}

\author{
V. G. ZVYAGIN, YU. E. GLIKLIKH, AND V. V. OBUKHOVSKII \\ Received 3 July 2006; Accepted 3 July 2006
}

Copyright (c) 2006 V. G. Zvyagin et al. This is an open access article distributed under the Creative Commons Attribution License, which permits unrestricted use, distribution, and reproduction in any medium, provided the original work is properly cited.

This issue contains the papers selected from the talks of the international conference "Topological and Variational Methods of Nonlinear Analysis and their Applications" dedicated to the 85th Jubilee of Professor A. D. Myshkis and the 75th Jubilee of Professor Yu. G. Borisovich. The conference took place in Voronezh, Russia, 27 June-2 July, 2005.

Professor Anatoly Dmitrievich Myshkis is the founder of a number of new scientific directions in the theory of functional differential equations (1949-1951), partial differential equations, differential inclusions and multivalued dynamical systems, and many others. In particular, he was one of the first researchers who studied retarded-type equations, he introduced the notion of a generalized solution for a differential equation with set-valued discontinuous right-hand side, his studies of set-valued maps with aspheric values (1954) found in the recent decades very important and interesting applications in the theory of differential equations, inclusions and control systems, and so forth. For the series of papers in the theory of set-valued differential equations, he was awarded a prize by the Moscow Mathematical Society.

In his activities, A. D. Myshkis pays special attention to the problems of applications. It is also worth pointing out his contribution to approximate and numerical methods, difference equations and inequalities, turbulent systems, impulse impact systems, spectral problems with variable boundary, and his analysis of the influence of velocity forces on oscillatory stability. A. D. Myshkis pays much attention to the methodology of applied mathematics and in his works he expressed his original views about how engineers and other specialists should be taught mathematics.

The perennial educational work of A. D. Myshkis prompted him to write several textbooks in mathematics and mathematical physics. These textbooks became very popular and were translated into many languages. 
A. D. Myshkis is a member of editorial boards of well-known international journals such as "Nonlinear Analysis: Theory, Methods and Applications," "Functional Differential Equations," and "Journal of Difference Equations and Applications."

Professor Yuri Grigorievich Borisovich is worldwide known by his works on topological methods in nonlinear analysis and their applications to mathematical physics, control theory, geometry, and many other branches of mathematics. First we should mention a series of his works in the 50th, and 60th, of twentieth century on relative topological degree and relative rotation of vector fields that yielded the development of the degree theory for weakly continuous mappings in Banach spaces, for condensing operators $(k$-set contractions), and applications to functional-differential equations, partial differential equations, and so forth.

In the series of works with his collaborators in the 70th, a new version of degree theory for nonlinear Fredholm mappings was suggested that allowed one to cover Fredholm mappings with compact and condensing perturbations. This theory got plenty of applications, first of all to partial differential equations.

Another topic, where Professor Borisovich's influence is very well known, is the theory of set-valued mappings and differential inclusions. A lot of research and survey papers (in particular, in Russian Mathematical Surveys) and two monographs (joint with B. Gel'man, A. D. Myshkis, and V. Obukhovskii; the last one in 2005) were published by him and his collaborators on this subject.

Borisovich's text book "Introduction to Topology" (joint with N. Bliznyakov, T. Fomenko, and Y. Izrailevich) has been translated into many foreign languages and is one of the best introductory books on this subject.

In this issue we include papers on the themes where the ideas of variational and topological methods are applied. Here A. D. Myshkis and Yu. G. Borisovich made significant input. It is some parts of topology, topological index theory, functional analysis, global analysis and analysis on manifolds, stochastic analysis, hydrodynamics, and so forth.

V. G. Zvyagin

Yu. E. Gliklikh

V. V. Obukhovskii 


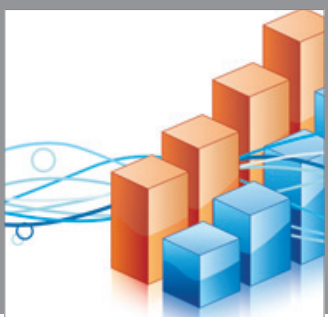

Advances in

Operations Research

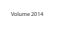

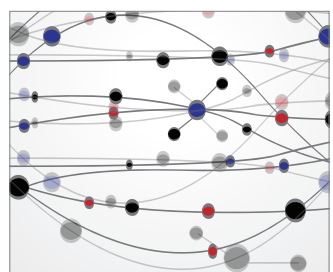

\section{The Scientific} World Journal
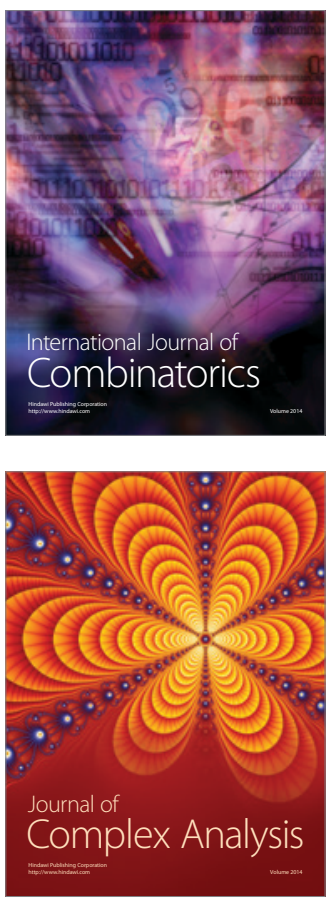

International Journal of

Mathematics and

Mathematical

Sciences
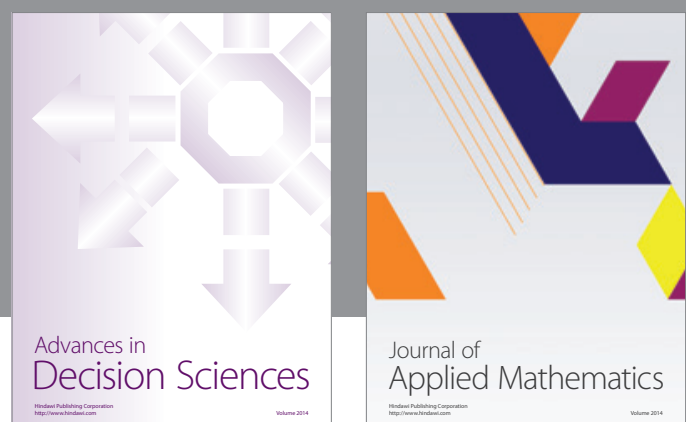

Journal of

Applied Mathematics
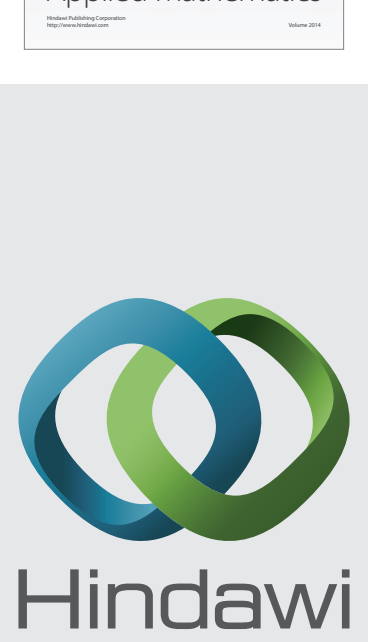

Submit your manuscripts at http://www.hindawi.com
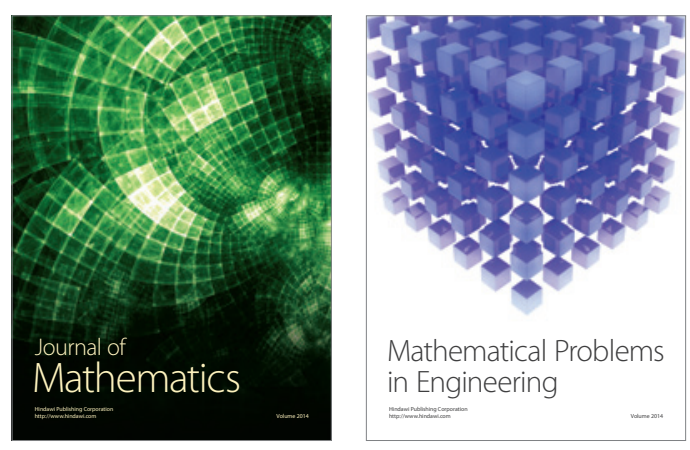

Mathematical Problems in Engineering
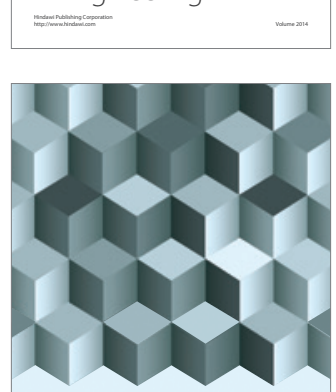

Journal of

Function Spaces
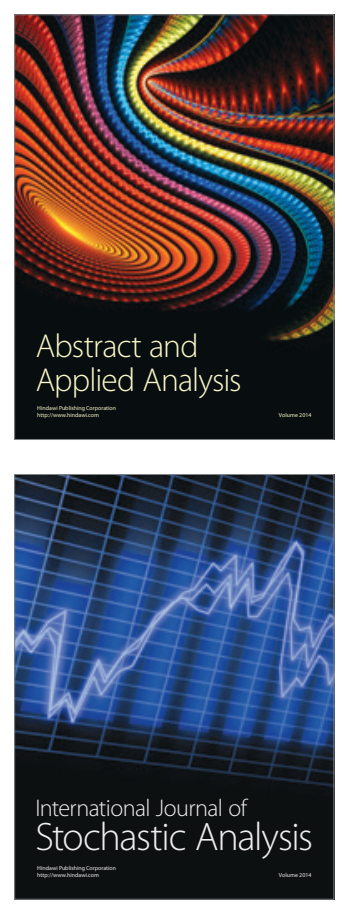

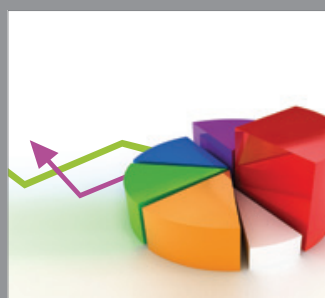

ournal of

Probability and Statistics

Promensencen
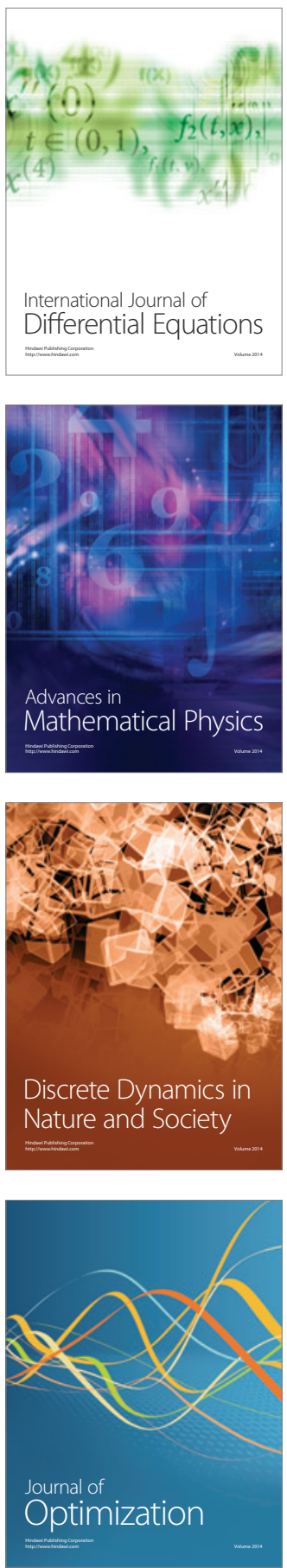